\title{
Persistent Primitive Hypoglossal Artery Associated With Multiple Cerebral Aneurysms
}

\author{
Rikako Yabuki ${ }^{a}$, Ei-ichi Baba ${ }^{\text {a }}$, Kazutaka Shirokane ${ }^{\mathrm{a}}$, Atsushi Tsuchiya ${ }^{\mathrm{b}}$, \\ Motohiro Nomura ${ }^{\mathrm{a}, \mathrm{c}}$
}

\begin{abstract}
Persistent primitive hypoglossal artery is a rare anastomosis between the carotid and basilar arteries, and sometimes associated with cerebral aneurysms. However, association of persistent primitive hypoglossal artery with aneurysms located on arteries other than persistent primitive hypoglossal artery itself or posterior circulation is very rare. An 80-year-old woman suffered from subarachnoid hemorrhage, whose angiography demonstrated aneurysms on the left middle cerebral artery and anterior communicating artery, and the left persistent primitive hypoglossal artery. The middle cerebral artery aneurysm was the origin of hemorrhage. Although repeated craniotomy was necessary for the left middle cerebral artery aneurysm, both aneurysms were successfully clipped. In our case, neither aneurysm was located on an artery related to the persistent primitive hypoglossal artery. There is a possibility that cases of persistent primitive hypoglossal artery are accompanied by cerebral aneurysms on arteries other than the persistent primitive hypoglossal artery or in the posterior circulation.
\end{abstract}

Keywords: Persistent primitive hypoglossal artery; Cerebral aneurysm; Subarachnoid hemorrhage; Middle cerebral artery; Anterior communicating artery

\section{Introduction}

Persistent primitive hypoglossal artery (PPHA) is the second most common anastomosis between the carotid and basilar arteries; and the incidence has been reported to be $0.03-0.26 \%$ [1]. The incidence of an association with a cerebral aneurysm has been reported to be as high as $22-26 \%[1,2]$. Recently, we treated a patient with PPHA who presented with subarachnoid hemorrhage (SAH). Radiological examinations revealed two cerebral aneurysms on the middle cerebral artery (MCA) and

Manuscript submitted October 6, 2018, accepted October 22, 2018

aDepartment of Neurosurgery, Kanto Rosai Hospital, Kawasaki, Japan ${ }^{b}$ Department of Neurology, Kanto Rosai Hospital, Kawasaki, Japan ${ }^{\mathrm{c} C}$ Corresponding Author: Motohiro Nomura, Department of Neurosurgery, Kanto Rosai Hospital, 1-1 Kizukisumiyoshi-cho, Nakahara-ku, Kawasaki 211-8510, Japan. Email: nomura413jp@yahoo.co.jp

doi: https://doi.org/10.14740/jocmr3649 anterior communicating artery (AComA) in addition to PPHA. In most cases of PPHA with cerebral aneurysms, the aneurysms are located on PPHA, the vertebrobasilar artery (VBA) or posterior circulation of the circle of Willis. In this report, we describe our experience of treatment for a patient with PPHA and multiple aneurysms, and discuss the radiological findings, management and etiology.

\section{Case Report}

An 80-year-old woman with a history of hypertension and angina pectoris experienced sudden-onset headache and vomiting. Computed tomography (CT) on admission demonstrated SAH (Fig. 1A), while three-dimensional CT angiography (3DCTA) demonstrated aneurysms of the left MCA and AComA. The sizes of the aneurysms of MCA and AComA were 9 and $3 \mathrm{~mm}$, respectively (Fig. 1B). Due to the shape and size, the left MCA aneurysm was suspected to be the origin of SAH. An anomalous artery originated from the left internal carotid artery (ICA) ran through the hypoglossal canal and connected to the basilar artery (BA) (Fig. 1C). Angiography also demonstrated the left PPHA (Fig.1D). In addition, a fenestrated thin artery was observed at AComA. On the day of onset, craniotomy was performed, and both aneurysms were clipped. Post-operative 3D-CTA revealed a small residual neck of the MCA aneurysm (Fig. 2A). The post-operative course was uneventful until the sixth day when the patient showed consciousness deterioration. CT revealed additional SAH in the basal cistern (Fig. 2B). Angiography demonstrated enlargement of the residual neck of the left MCA aneurysm (Fig. 2C). Repeated operation was performed. Recurrence of the aneurysm was noted at the aneurysmal neck, and it was re-clipped. Post-operative 3D-CTA demonstrated no residual aneurysm on the left MCA (Fig. 2D). After the second operation, no symptom due to vasospasm was observed. On the 34th day, right ventriculo-peritoneal shunt was performed. On the 88th day, she was transferred to another hospital for rehabilitation.

\section{Discussion}

Although PPHA is a rare congenital anomaly, the incidence of its association with cerebral aneurysms is high [2]. Among them, $31.4 \%$ of the aneurysms are located on PPHA itself and $53 \%$ on the posterior part of the circle of Willis [3]. There have 

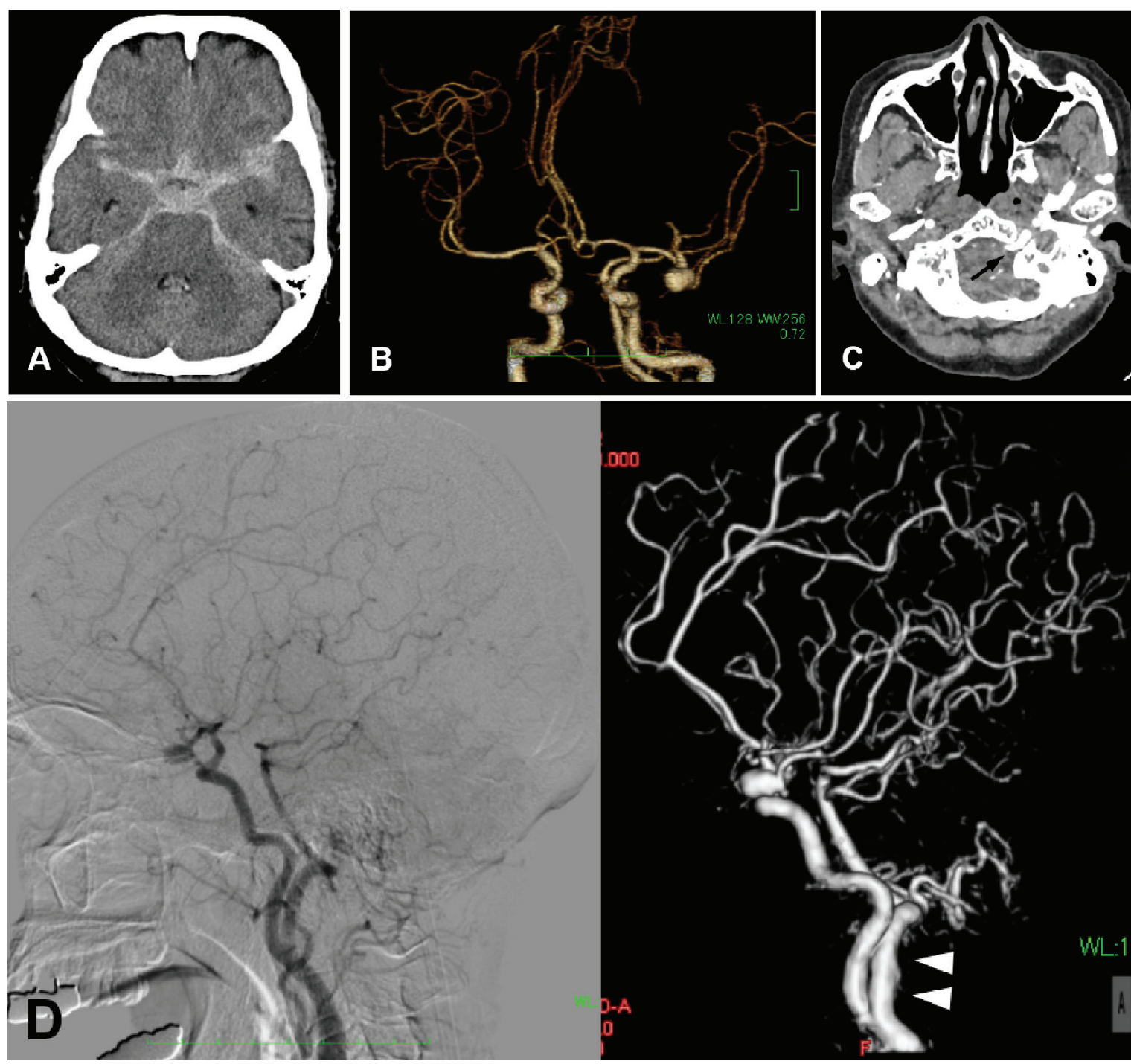

Figure 1. (A) CT demonstrating SAH and enlargement of the ventricles. (B) 3D-CTA demonstrating two aneurysms at AComA and the left MCA. The left MCA aneurysm projects medially. (C) A raw image of CTA showing an artery running through the left hypoglossal canal (arrow). (D) Angiography (left: conventional, right: 3D) demonstrating PPHA (arrowheads) originating from the left ICA.

been some reports describing the association of PPHA with multiple aneurysms. Ikeda et al summarized cases of PPHA with multiple aneurysms in the Japanese literature [4]. According to them, nine cases of PPHA associated with multiple aneurysms have been reported [5-12]. Among them, eight cases had at least one aneurysm on PPHA or an artery in the posterior circulation. Only one case had aneurysms that were not related to PPHA or VBA [7]. Our patient had two aneurysms of arteries in the anterior circulation, on MCA and AComA. This is the second case of PPHA associated with aneurysms on arteries not related to PPHA or VBA. Although it is rare, we should be aware of the association of PPHA and cerebral aneurysms other than in the posterior circulation.

Increased hemodynamic stress of PPHA might lead to the formation of cerebral aneurysms, because PPHA is the main vessel supplying blood flow to the posterior circulation [13].
The most common location of an aneurysm in cases with PPHA is the intracranial portion of PPHA [13]. In our case, aneurysms were located on MCA and AComA. Although the aneurysms were not related to PPHA, there is a possibility that the hemodynamics was unusual due to the existence of PPHA, and aneurysms were formed in arteries other than PPHA. In our case, the posterior circulation was mainly fed by the left PPHA. The right posterior communicating artery (PComA) was of the fetal type, and the right posterior cerebral artery (PCA) was fed via the right PComA. PPHA originated from the left ICA. Therefore, the left ICA supplied blood flow to the left anterior circulation and posterior circulation other than territory of the right PCA. This condition required abundant blood flow to the left ICA, resulting in hemodynamic stress loaded on the left ICA system, and the aneurysms were formed. 

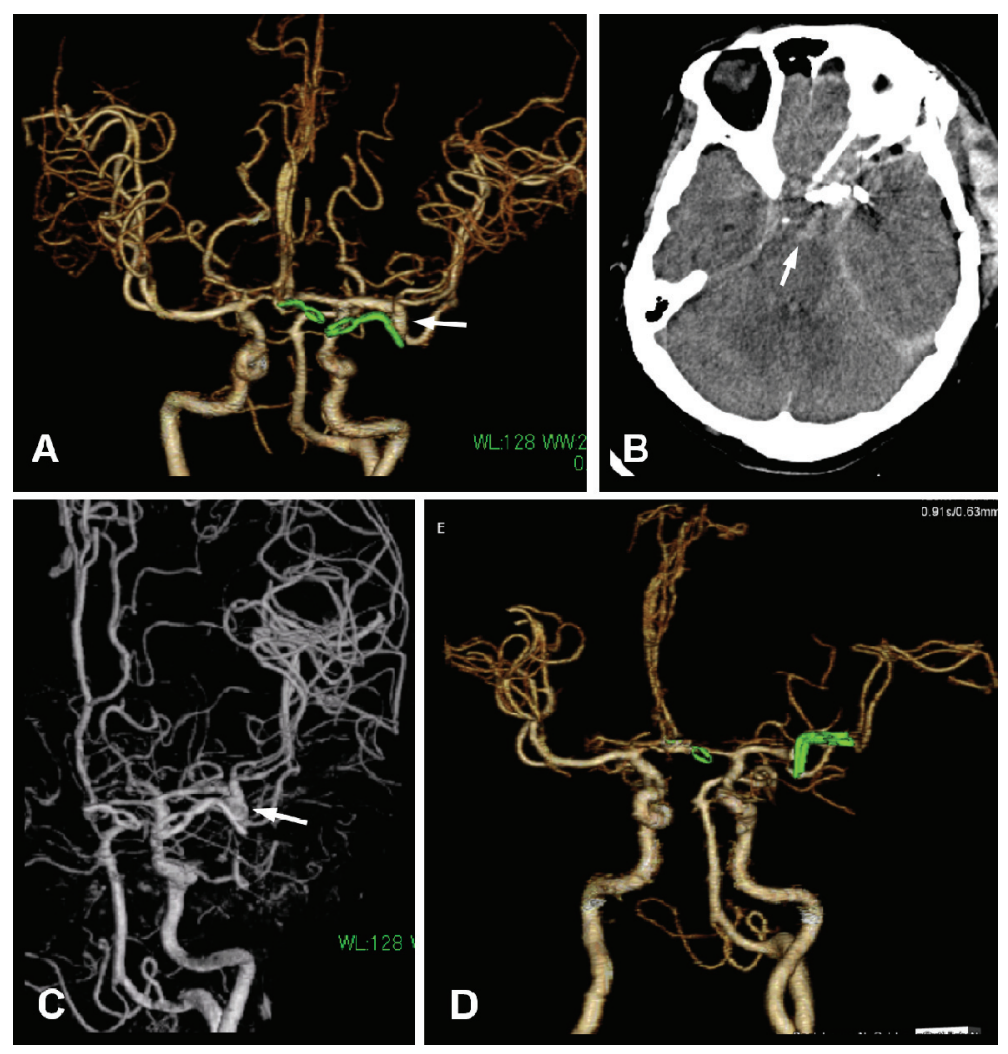

Figure 2. (A) Post-operative 3D-CTA demonstrating a small residual neck of the MCA aneurysm (arrow). (B) CT on the sixth day showing additional hemorrhage in the basal cistern (arrow). (C) Angiography showing enlargement of the residual neck of the MCA aneurysm (arrow). (D) After the second operation, the aneurysm is not observed on 3D-CTA.

\section{Conclusions}

In cases of PPHA, the incidence of an association with cerebral aneurysms is high. Most cases have aneurysms on PPHA or arteries in the posterior part of the circle of Willis. However, there is a possibility that cases of PPHA are accompanied by cerebral aneurysms in the anterior circulation.

\section{Acknowledgments}

This study was partly supported by research funds to promote the hospital functions of Japan Organization of Occupational Health and Safety.

\section{Conflict of Interest}

None.

\section{References}

1. Agnoli AL. Vascular anomalies and subarachnoid haemorrhage associated with persisting embryonic vessels. Acta Neurochir (Wien). 1982;60(3-4):183-199.
2. Yamanaka K, Noguchi K, Hayasaki K, Matsuoka Y. Persistent primitive hypoglossal artery associated with arteriovenous malformation - case report. Neurol Med Chir (Tokyo). 1990;30(12):949-955.

3. Kanematsu M, Satoh K, Nakajima N, Hamazaki F, Nagahiro S. Ruptured aneurysm arising from a basilar artery fenestration and associated with a persistent primitive hypoglossal artery. Case report and review of the literature. J Neurosurg. 2004;101(3):532-535.

4. Ikeda N, Nishizaki T, Sakakura T, Fujii N. A case of multiple unruptured cerebral aneurysms associated with persistent primitive hypoglossal artery. JNET. 2015;9:266271, in Japanese.

5. Bohmfalk GL, Story JL. Aneurysms of the persistent hypoglossal artery. Neurosurgery. 1977;1(3):291-296.

6. Duffill J, Lang DA, Dwyer GN. Subarachnoid haemorrhage in a child from an aneurysm of a persistent primitive hypoglossal artery. Br J Neurosurg. 1996;10(6):607610.

7. Fujii Y, Arai H, Takeuchi S, Sasaki O, Kamada K, Ogawa $\mathrm{H}$, Shimbo Y. [An autopsy case of persistent primitive hypoglossal artery with multiple cerebral aneurysms]. No Shinkei Geka. 1988;16(4):421-426, in Japanese.

8. Kobayashi H, Munemoto S, Hayashi M, Yamamoto S. Association of persistent hypoglossal artery, multiple intracranial aneurysms, and polycystic disease. Surg Neurol. 1984;21(3):258-260. 
9. Murayama Y, Fujimoto N, Matsumoto K. Bilateral persistent primitive hypoglossal arteries associated with a large ruptured aneurysm on one side. Surg Neurol. 1985;24(5):498-502.

10. Nishimura H, Uemura Y. Multiple cerebral aneurysms associated with persistent primitive hypoglossal artery: case report. Jpn J Neurosurg (Tokyo). 2001;10:481-486, in Japanese.

11. Teo M, Bhattacharya J, Suttner N. Persistent hypoglossal artery - an increased risk for intracranial aneurysms? Br J Neurosurg. 2012;26(6):891-892.

12. Waga S, Morooka Y, Kojima T. Aneurysm on a persistent hypoglossal artery. Acta Neurochir (Wien). 1981;59(12):71-78.

13. Kimball D, Ples H, Miclaus GD, Matusz P, Loukas M. Persistent hypoglossal artery aneurysm located in the hypoglossal canal with associated subarachnoid hemorrhage. Surg Radiol Anat. 2015;37(2):205-209. 